\title{
EXAMPLES OF MICROSTRUCTURE-RELATED PROPERTIES OF GALLIUM NITRIDE
}

\author{
M. LESZCZYŃSKI* \\ High Pressure Research Center Unipress, Polish Academy of Sciences \\ Sokołowska 29/37, 01-142 Warsaw, Poland
}

The work provides a brief overview and the latest experimental results concerning the microstructure of gallium nitride. Because of the importance for the optoelectronic and electronic technologies, mainly problems related to the lattice mismatch between substrates and GaN layers are discussed. Three main substrates, sapphire, silicon carbide and high-pressure-grown bulk GaN crystals, are compared. Mosaicity, thermal strains and surface roughnesses of the GaN layers grown on those substrates are reported. The application of high-pressure technologies makes it possible to use temperatures higher by a few hundred degrees with respect to the atmospheric pressure for which the decomposition of gallium nitride occurs at temperatures below $1000^{\circ} \mathrm{C}$. Annealing at pressures higher than $10 \mathrm{kbar}$ and temperatures up to $1550^{\circ} \mathrm{C}$ causes modifications of the microstructure of $\mathrm{GaN}$ heteroepitaxial layers on sapphire. For example, their mosaicity decreases as observed by narrowing of the X-ray diffraction peaks. The implanted layers recover upon high-pressure annealing and give a strong dopant-related luminescence.

PACS numbers: $61.72 . \mathrm{Ji}, 61.72 . \mathrm{Vv}$

\section{Introduction}

Gallium nitride and its solid solutions with $\mathrm{InN}$ and $\mathrm{AlN}$ are direct-wide-band-gap semiconductors covering the green/blue/UV range of the spectrum. Due to the commercial successes of the blue light-emitting diodes (LEDs) and the first c.w. blue laser operating for more than $30 \mathrm{~h}$ at room temperature (both constructed by Nakamura, Ref. $[1,2])$ the nitrides experience a top popularity. However, in comparison with other semiconductors, many of their physical properties are still not well known. Such a situation arises from the fact that the research is done mainly on heteroepitaxial layers grown on highly mismatched substrates, sapphire and silicon carbide. Mismatch-related defects of high concentrations make it hardly possible to measure many of the GaN features and also decrease the efficiencies of optoelectronic and electronic devices. The growth of GaN single crystals is difficult as this material at $1 \mathrm{~atm}$ of nitrogen decomposes at temperatures below $1000^{\circ} \mathrm{C}$,

*e-mail: mike@iris.unipress.waw.pl 
whereas the melting point is of about $2500^{\circ} \mathrm{C}$ at pressure of about $45^{\circ} \mathrm{kbar}$ (Ref. [3]). In order to increase the available temperature range, high pressure of nitrogen must be applied. It is an approach of Unipress, where high pressure is used for growth of the unique single crystals of $\mathrm{GaN}$ and for annealing the heteroepitaxial layers.

The presented paper gives a short overview of the research done in Unipress on GaN microstructure and its influence on some of the optical properties.

\section{Substrates for GaN planar technology}

\subsection{Lattice mismatch}

The problem of critical conditions for lattice relaxation (by emitting misfit dislocations) of heteroepitaxial layers is very complex, as one should take into account not only lattice mismatch and layer thickness, but also substrate roughness, density of defects, growth mode of the layer, interaction of defects, etc. However, for the needs of this paper, Fig. 1 shows critical thicknesses and mismatches for the best known system $\mathrm{Si}-\mathrm{Ge}$ (elastic properties similar to $\mathrm{GaN}$ ) together with the first experimental results for nitrides.

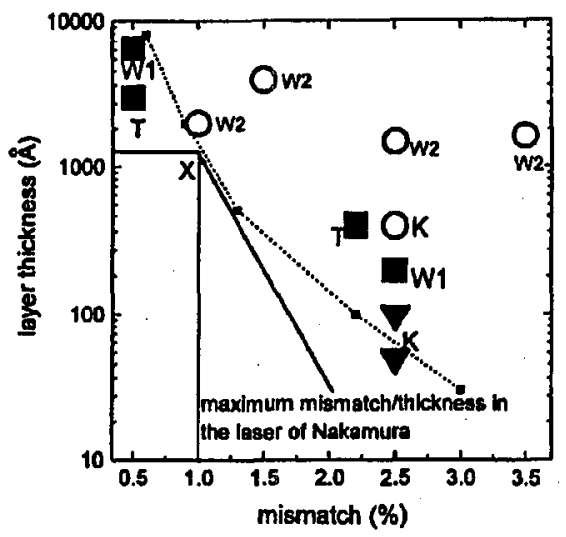

Fig. 1. Critical thickness for the nitride layers versus their thickness. Open circles W2 - fully relaxed, this work, MOCVD-grown AlGaN layers on SiC; full squares - fully strained; W1 - this work, MBE-grown AlGaN layers on the bulk GaN; T - Takeuchi et al., Ref. [11], MOCVD-grown AlGaN and InGaN layers on GaN on sapphire; full triangles - partially relaxed, MOCVD-grown AlGaN layers on GaN on sapphire; $\mathrm{K}$ Kim et al., Ref. [12]; the dotted line denotes experimental results for $\mathrm{Si-Ge} \mathrm{system.}$

It can be seen that for the most popular substrate, sapphire ( $16 \%$ lattice mismatch to $\mathrm{GaN}$ ), the relaxation should occur after depositing the first monolayer. In the case of silicon carbide ( $3.5 \%$ mismatch to $\mathrm{GaN})$, the GaN layer should start relaxing at a thickness of a few tens of angstroms.

The question can be asked if the GaN bulk crystals are potential substrates to grow a fully strained blue-laser structure. To answer this question the Nichia laser structure can be analysed. In Fig. 1 the most critical point for relaxation denoted by the letter $\mathrm{X}$ is shown (higher, $2 \%$ mismatch to $\mathrm{GaN}$ is in the quantum wells 
of InGaN, but they are very thin). It can be seen that most probably the Nichia laser structure grown on the GaN bulk crystal would not be relaxed. Additionally, it should be possible to construct the laser structure with thinner layers if the density of dislocations was smaller.

However, the bulk crystals are not yet commercially a vailable and the layers on sapphire (about $10^{9}-10^{10} \mathrm{~cm}^{-2}$ dislocations) and on SiC (about $10^{7}-10^{9} \mathrm{~cm}^{-2}$ dislocations) are the most extensively examined. The quality of these layers depends very strongly on the thickness of the buffer layers of AlN or GaN which grow amorphous at low temperatures and then crystallize at higher temperatures.

\subsection{Comparison of crystallographic quality of GaN layers grown on various substrates}

The most popular method of evaluating the crystallographic quality of the samples is X-ray diffraction. The typical FWIIM (full width at half maximum) of $00.2 \mathrm{Cu} K_{\alpha 1}$ Bragg peaks (rocking curves) are of 100-1000 arc sec for GaN layers grown on sapphire and 40-250 arc sec for layers grown on $\mathrm{SiC}$ (both (00.1) oriented). For the homoepitaxial layers the FWHM are similar to those for the bulk GaN substrates. At present, the best bulk crystals are those grown at high pressure. They possess a nearly perfect structure (20 arc sec of the rocking curves), but usually the samples of a size up to $10 \mathrm{~mm}$ consist of a few crystallites of a size $0.2-5 \mathrm{~mm}$ misoriented by $0.1-1$ arc min with respect to each other (Ref. [4]). Mg-doped crystals are even of a higher quality as they do not have strains related to the inhomogeneities of free-electrons distribution. The largest difference between $\mathrm{GaN}$ layers on sapphire or $\mathrm{SiC}$ and homoepitaxial layers, is in the in-plane direction. This direction is not usually explored using $\mathrm{X}$-ray diffraction as for this purpose a very intense X-ray beam must be used. Recent measurements showed

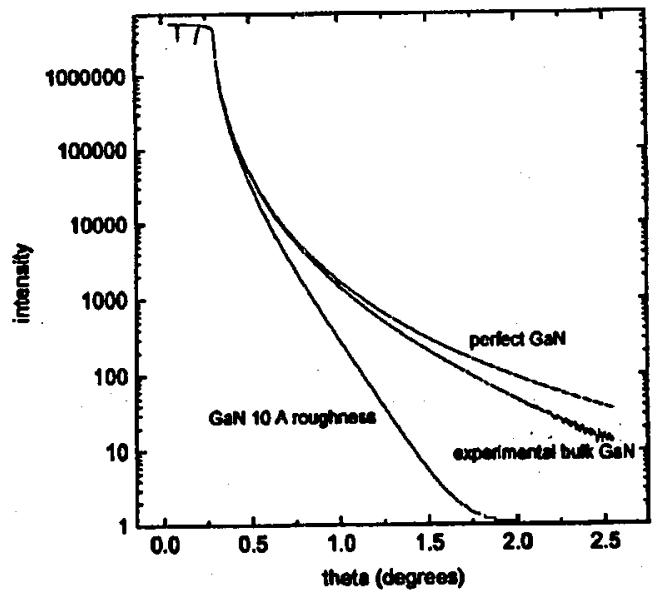

Fig. 2. X-ray reflectivity curve for the chemically polished GaN crystal. The theoretical curves for a perfect and $10 \AA$ rough surface are given for comparison. 
that the FWHM of 22.0 reflection is of 20 arc sec for the bulk crystal and deposited homoepitaxial layer, whereas for the good GaN layer on sapphire is of about 0.4 degrees (only 180 arc sec for 00.2 reflection).

The other important microstructural parameter is the roughness of the substrate and the growing layer, crucial for constructing quantum structures. The roughness is not only a result of a non-optimised growth, but also is related to the relaxation process and the 3D growth mode. Figure 2 shows the X-ray reflectivity curves for the state-of-art GaN layers on sapphire of different thicknesses and homoepitaxial GaN layer grown on the chemically polished bulk crystal. Chemical polishing of $\mathrm{GaN}$ nitrogen-terminated side has recently been developed by Weyher et al. (Ref. [5]). The procedure gives an atomically flat surface as was confirmed not only by X-ray reflectivity (long-range smoothness), but also by atomic-force microscopy (nanometer resolution).

\subsection{Thermal strain}

Lattice relaxation occurs at elevated temperatures. At lower temperatures, during cooling, thermal strain can be created if the thermal expansions of a layer and of a substrate are different. For high quality heteroepitaxial layers (for example, $\mathrm{GaAs}$ on $\mathrm{Si}$ ), the thermal strain corresponds to the one calculated using the thermal expansion coefficients (TECs) of the layer and of the substrate. For GaN on sapphire and on $\mathrm{SiC}$ the situation is more complex. For GaN on sapphire, the layers are always in-plane compressed, however, usually much less than one could expect from the TECs for those two materials. Most probably, this phenomenon is related to the microstructure of these GaN layers which are not uniform and grains may slide with respect to each other, rather than to follow the dimensions of the substrate. Thus, a thermal strain should be larger for higher quality samples. For $\mathrm{GaN}$ on $\mathrm{SiC}$, both the compressive and tensile strains have been reported. As these layers are of a good crystallographic quality, the compressive strain is presumably a result of a non-full relaxation of the layer (thermal strain is a tensile one).

A problem, which is not solved yet, is how to calculate the relaxed lattice parameters, which may vary depending, for example, on the level of doping, from the measured parameters for the strained layers. The following formula:

$$
(\Delta a / a) /(\Delta c / c)=(\nu-1) / 2 \nu
$$

describes the relative change of lattice parameter $c$ perpendicular to the surface induced by the change of lattice parameter $a$ parallel to the surface. The Poisson coefficient $\nu$ reported so far ranges from 0.20 (Ref. [6]) to 0.37 (Ref. [7]). From our experiments, we recommend to use the value of 0.25 .

Discussing the expected thermal strains one should be aware that TECs of any semiconductor depend on many parameters (doping level, defect concentration, etc.) and may vary even by $25 \%$. An example of how thermal expansion increases with free-electron concentration is given in Fig. 3. This figure shows an increased difference between lattice parameters of the bulk crystal $\left(5 \times 10^{19} \mathrm{~cm}^{-3}\right.$ of free electrons) and the homoepitaxial layer which can be treated as a standard material of gallium nitride. 


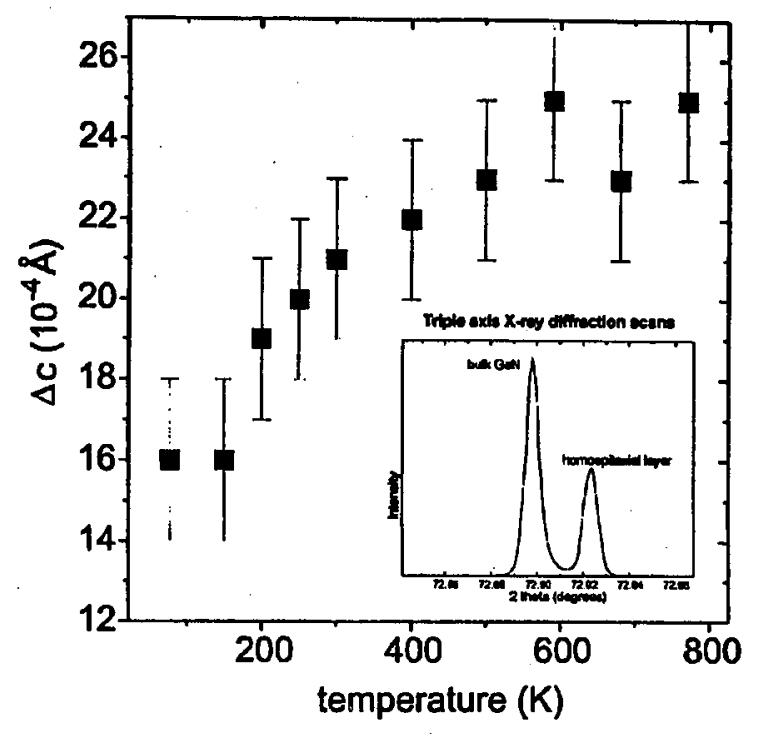

Fig. 3. Temperature dependence of the difference in lattice parameters between the bulk GaN sample of a high free-electron concentration and a homoepitaxial layer treated as a standard. The inset illustrates the separation of the Bragg peaks from the GaN substrate and the layer.

Doping changes the lattice parameters not only by a hydrostatic expansion or contraction, but also changes the Poisson coefficient. For the most popular $p$-dopant, magnesium, its concentration of about $10^{20} \mathrm{~cm}^{-3}$ increases lattice parameters by $\Delta a=0.006 \AA$ and $\Delta c=0.009 \AA$ (despite the smaller ionic radius of magnesium with respect to gallium). For silicon, which is a main $n$-type dopant, its concentration of about $10^{19} \mathrm{~cm}^{-3}$ caused the following change of lattice parameters: $\Delta a=0.0001 \AA, \Delta c=0.0006 \AA$. Again, just using Vegard's law one would rather predict lattice contraction, because the silicon ion is smaller than the gallium one. In this case, the increase in lattice parameters can be caused by free electrons via the deformation potential of the conduction band (Ref. [8]).

\section{Effects of annealing on properties of GaN heteroepitaxial layers}

As mentioned in the introduction, $\mathrm{GaN}$ decomposes at temperatures about two and a half times smaller than the melting point. For other semiconductors, an empirical law predicts that the structural defects become mobile at temperatures larger than about sixty percent of the melting point. In order to extend the available annealing temperature range, we applied high pressure of nitrogen.

\subsection{Changes of mosaicity caused by annealing}

Figure 4 shows the dependence of the FWHM of the X-ray rocking curves for one of the examined GaN layer on sapphire versus annealing temperature. It can be seen that the changes are very significant at temperatures above about 

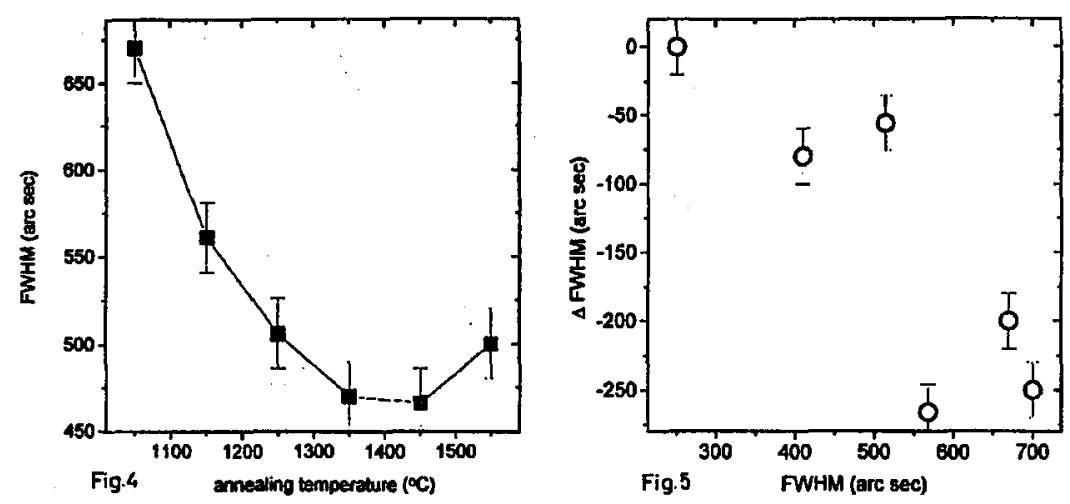

Fig. 4. Half-width of X-ray rocking curve for GaN layer on sapphire versus annealing temperature at a high pressure of $10 \mathrm{kbar}$.

Fig. 5. Change of the half-width of the X-ray rocking curves for various GaN layers on sapphire after annealing at $1350^{\circ} \mathrm{C}$ and $10 \mathrm{kbar}$ for $1 \mathrm{~h}$.

$1200^{\circ} \mathrm{C}$. Figure 5 presents the changes of FWIIM for various samples grown in various laboratories. All samples were annealed for the same time (1 hour) and at the same conditions $\left(1350^{\circ} \mathrm{C}, 10 \mathrm{kbar}\right)$.

\subsection{Changes of thermal strain caused by annealing}

Figure 6 shows the lattice parameters of one of the samples versus annealing temperature. It can be seen that this dependence is qualitatively similar to Fig. 4 . The increased thermal strain after annealing at temperatures above $1200^{\circ} \mathrm{C}$ cannot be explained just by using temperature higher than the growth temperature $\left(1050^{\circ} \mathrm{C}\right)$. If it were so, differences would be observed between samples annealed at $1350^{\circ} \mathrm{C}$ and $1550^{\circ} \mathrm{C}$, similar to the differences for the samples annealed at $1150^{\circ} \mathrm{C}$ and $1550^{\circ} \mathrm{C}$. Therefore, the observed increase in the thermal strain is rather related to the mosaicity decrease.

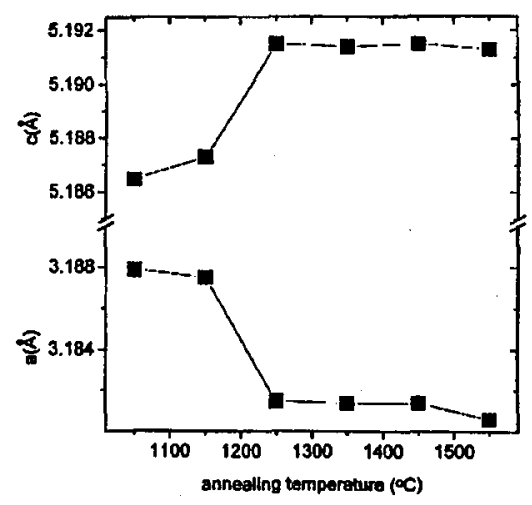

Fig. 6. Thermal strain of GaN layer on sapphire versus annealing temperature. 


\subsection{Post-implantation annealing}

One of the most popular way of introducing the dopants into semiconductors is ion implantation. For gallium nitride, this method has been reported by a number of authors. The most spectacular results were obtained by Zolper et al. (Ref. [9]), who applied post-implantation rapid thermal annealing (RTA) at temperatures up to $1150^{\circ} \mathrm{C}$. The annealing removed some of the implantation defects and both, $n$-type and $p$-type GaN layers on sapphire were produced.

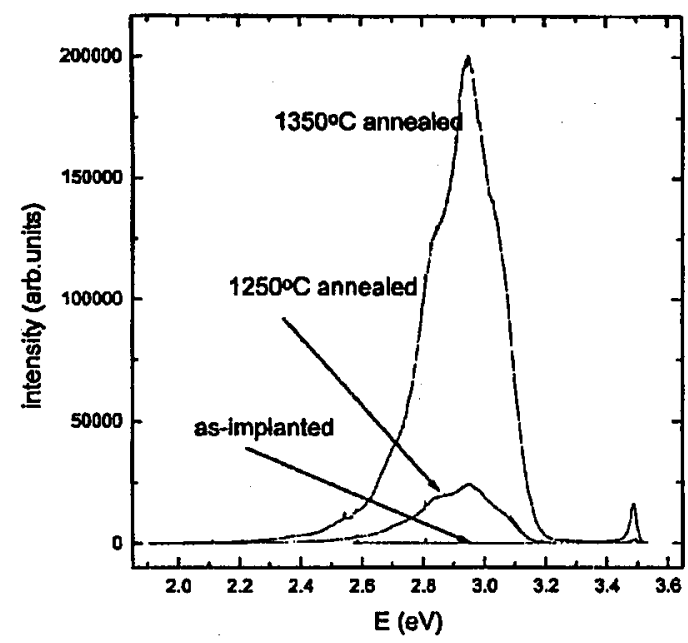

Fig. 7. Photoluminescence spectra for $\mathrm{Zn}$-implanted $\mathrm{GaN}$ layers on sapphire after annealing at high pressure.

In the research carried on in Unipress, we examined GaN heteroepitaxial layers implanted with $\mathrm{Zn}$. Figure 7 shows the photoluminescence spectra for different temperatures of annealing. It can be seen that a temperature of about $1350^{\circ} \mathrm{C}$ is the most efficient for having an intense PL signal. This fact is in correspondence with the improvement of crystallographic quality reported in the previous sections. At higher temperatures, it is possible that new defects are created and/or some impurities are diffused-in during the annealing procedure at nitrogen atmosphere.

\section{Discussion}

The first part of the paper dealt with three main substrates for GaN epitaxy, sapphire, SiC and the bulk GaN crystals. The most mature technology is the one using sapphire and buffer layers of GaN or AlN grown at low temperatures. Despite a very high density of dislocations, devices based on that technology produce a very intense light and are characterised by a long life-length. It is presumably due to a low mobility of dislocations and a certain localization of regions emitting light, for example, due to a non-homogeneous distribution of indium in the quantum wells. However, even if dislocations do not act as non-radiative centers, they scatter light, thus diminishing the intensity of emitted light. SiC substrates offer a much 
better crystallographic quality of the $\mathrm{GaN}$ layers. However, $\mathrm{SiC}$ crystals have their disadvantages as extended defects or not epi-ready surfaces.

The single crystals of gallium nitride grown at extreme conditions of pressure and temperature are, at present, of a size up to $10 \mathrm{~mm}$ and of a very low density of extended defects. An epi-ready surface obtained by chemical polishing, very good thermal and electrical conductivity make these substrates very attractive for constructing the top-effective devices. Recently, the group of M. Kamp of the Ulm University constructed a light-emitting diode based on homo- $p-n$ junction (Ref. [10]). The LED is about twice brighter with respect to the analogous one constructed using sapphire as a substrate.

The second part of the paper dealt with the application of high-pressure annealing for changing the microstructure of heteroepitaxial layers. The first experimental results are very promising. It is possible to decrease the mosaicity of the layers, as well as to make a recovery of the layer damaged by implantation. However, for some of the layers, yellow luminescence significantly increased after an annealing process. This luminescence is related to the presence of native defects (presumably, gallium vacancies). An additional phenomenon observed for the annealed samples is a high diffusion rate of dopant atoms for heteroepitaxial layers. It creates a possibility of introducing dopants just by annealing, but has a detrimental effect if abrupt interfaces are to be created. The high diffusion rates may be related to a high concentration of dislocations in the heteroepitaxial layers. Further studies will be performed to understand the changes of layer microstructure and to optimise the annealing conditions.

\section{Acknowledgments}

The author is indebted to all his co-workers, who contributed to this work: J. Domagała, I. Grzegory, P. Porowski, P. Prystawko, T. Suski, A. Śliwiński, T. Strite and H. Teisseyre. Some of the work was sponsored by the grant of the Committee for Scientific Research (Poland) no. 175/P03/96/10.

\section{References}

[1] S. Nakamura, M. Senoh, N. Iwasa, S. Nagahama, T. Yamada, T. Mukai, Jpn. J. Appl. Phys. 2, Lett. 34, L1332 (1995).

[2] S. Nakamura, M. Senoh, S. Nagahama, N. Iwasa, T. Yamada, T. Matsushita, Y. Sugimoto, H. Kiyoku, Appl. Phys. Lett. 70, 868 (1996).

[3] J.A. Van Vechten, Phys. Rev. B 7, 1479 (1991).

[4] M. Leszczyński, I. Grzegory, H. Teisseyre, T. Suski, M. Boćkowski, J. Jun, J.M. Baranowski, S. Porowski, J. Domagala, J. Cryst. Growth, in press.

[5] J. Weyher, I. Grzegory, S. Porowski, unpublished.

[6] T. Azuhata, T. Sota, K. Suzuki, J. Phys., Condens. Mater. 8, 3111 (1996).

[7] V.A. Savestanco, V.U. Shelag, Phys. Status Solidi A 48, K135 (1978).

[8] T. Figielski, Phys. Status Solidi 1, 306 (1961).

[9] J.C. Zolper, M. Hagerott Crawford, S.J. Pearton, C.R. Abernathy, C.B. Vartuli, C. Yuan, R.A. Stall, J. Electron. Mater. 25, 839 (1996). 
[10] M. Kamp, C. Kirchner, M. Mayer, A. Pelzmann, M. Leszczyński, I. Grzegory, S. Porowski, unpublished.

[11] T. Takeuchi, H. Takeuchi, S. Sota, H. Sakai, H. Amano, I. Akasaki, Jpn. J. Appl. Phys. 36, L177 (1997).

[12] C. Kim, I.K. Robinson, J. Myoung, K. Shim, M.-C. Yoo, K. Kim, Appl. Phys. Lett. 69, 2358 (1996). 